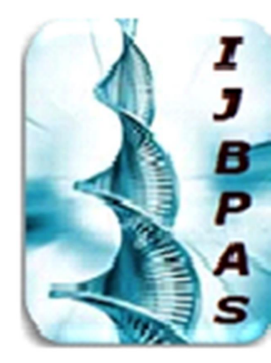

International Journal of Biology, Pharmacy and Allied Seiences (IJBPAS) 'A Bridge Betusen Caboratory and QRendo'

Www.ijbpas.com

\title{
APPROACH TO THE GENETIC ALGORITHM FOR SEGMENTING AND ANALYSING MEDICAL IMAGES
}

\section{JEYABHARATHI. $\mathrm{J}^{1^{*}}$, PRAVEENA G.N ${ }^{2}$, SINDHUJA $\mathbf{V}^{3}$, SREEVANI.M ${ }^{4}$, RANJAN WALIA $^{5}$ AND BADUGU SURESH ${ }^{6}$}

1. Associate Professor in Computer Science and Engineering at Koneru Lakshmaiah Education Foundation, Greenfields, Vaddeswaram, Guntur

2. Associate Professor in Electronics and Communication Engineering at R.M.K. College of Engineering and Technology, Kavaraipettai, Tamil Nadu, India

3. UG Student, Final Year in Electrical and Electronics Engineering at KPR Institute of Engineering and Technology, Coimbatore

4. Assistant Professor in Electronics and Communications Engineering at Institute of Aeronautical Engineering, Hyderabad, Telangana

5. Associate Professor in Electrical Engineering at Model Institute of Engineering and Technology, Jammu J\&K, India

6. Associate Professor in Electronics and Communication Engineering at Koneru Lakshmaih Education Foundation, Vadeswram, Guntur, Andhar Pradesh *Corresponding Author: Jeyabharathi. J; E Mail: shyambharathi2006@gmail.com

Received $20^{\text {th }}$ July 2021; Revised 22 ${ }^{\text {nd }}$ Aug. 2021; Accepted $30^{\text {th }}$ Sept. 2021; Available online $1^{\text {st }}$ Nov. 2021 https://doi.org/10.31032/IJBPAS/2021/10.11.1061

ABSTRACT

Ultrasound is required for producing medical pictures of the mammalian anatomy. Diagnostics imaging had represented the humongous leap further by embracing the workplace on picture capture. Image detection was used to combine the vast bulk of medical information contained in photos taken at different temporal periods and from diverse perspectives. For the study, 2 image methods are enrolled using a density true sequence approach. So, each angle of 
the shot is rotated, and this approach is evaluated for performance in order to demonstrate the product's efficacy. Its method likewise includes analyzing the 2 elements and, based upon its results, selecting a best measurement for an evolving process.

\section{Keywords: Picture registrations, evolutionary method, and resemblance meter}

\section{INTRODUCTION}

This content in pictures is aligned and visualized as an appearing via template matching. A Recognition system works in a variety of situations, including photographs collected at different times, with different points of view, and/or with various sensors [1]. Picture registration's purpose is to determine the optimal geometry mappings for aligning the pictures [2-3]. Diagnoses, plans of care, computer-assisted treatments, as well as biomedicine are all benefiting from this geometrical matching of medical data. There are two types of image enrollment: voxel or intensity-based \& feature-based. This full picture is used in an intensity-based technique, whereby researchers work exclusively on pixel intensities. Characteristics such as contouring, crested ridges, and vertices must be taken from pictures in the pre-processing phase and compared in the feature-based technique [4].

Poor signal-to-noise ratios, high information dimensionality, plus substantial variation in the distribution of the data are common traits of clinical skeletal images [5]. Furthermore, the brain cancer picture does not have any fixed actual size [6]. Because the grey values of lesion tissues or normal tissues might coincide, the surrounding normal area may appear aberrant. As a result, the excellent separation of biological skeletal images and brain tumor images is a vital step in locating small alterations and illness abnormalities. A superpixel segmentation technique [7] separates two-dimensional (2D) / three-dimensional (3D) picture elements onto chunks based on color, structure, as well as other characteristics. The picture's localized characteristics or operational structural components can be represented by the blocks. Furthermore, based on the chosen weighting, the spectra could refer to the number and location of the original initial centroids. This superpixel segmentation technique could significantly decrease computing redundancies as well as huge device throughput time, produce regular forms, get the precise spatial correlation, and also have limited performance [8].

\section{Related Works}

The scientific method converts that picture together into the structure and then 
separates the image information using graphtheoretical expertise. Every vertex inside the network correlates to a pixel location, and the boundary is generally used to weigh overall similarities between documents adjacent routers when expressing the picture as an unordered list containing scores. This graphbased technique and the normalized cutting method are two examples of traditional graph theory [9]. That similarity measurement among superpixels is being used by the gradients raising method to partition the picture onto distinct areas. This disparity between both the spots as well as the resemblance of the characteristics is repeated several times by altering the amount and position of the seed funding elements. A restriction is created for each pixel with the same properties [10].

The intensity-based technique outperforms the functionality technique for picture recognition [11]. Multiple factors influence object recognition approaches, including the kind of registrations (external or internal), the transformations paradigm, as well as the optimization procedure [12]. The most widely used techniques for healthcare image acquisition are predicated upon voxelbased similarity metrics that are optimized. Surfaces registrations approaches focus on 3 variables: transition selection, resemblance requirement, \& universal optimization [13]. Regarding intensity-based clinical picture registrations, various measurements including such sum of squared variations, the sum of absolute differences, data fusion, and standardized correlations could be employed [14]. Evolutionary algorithms could address the limitations of classic numerical-based optimization techniques such as backpropagation and Powell's approach. It escapes global optimum by finding an optimal result [15].

Machine learning, which essentially utilizes a computer's understanding to imitate brains neurotransmitters and analyze MRI scans, is indeed a potential method to create a powerful assisted healthcare diagnostic \& therapy device. In [16], for contrast, a novel method integrating randomized forests plus superpixel identification is presented. That technique employs Lagrange's approximation over overlapped arcs area produced by convex methods and theory to retrieve the fine edge information. That system is preferable to preserving the border characteristics of normal tumors. Furthermore, it uses convex recognition to discriminate between the real overlapped zone as well as the projected ones. However, whenever it comes to fragmenting various 
types of complicated medical pictures, the system has to be enhanced.

The optimization algorithm is used to register images throughout this paper. The most important stage in GA is deciding on the optimization problem which will be used to assess every chromosome's efficiency. These resemblance measurements, which would be the target value, establish the strength value. The GA method uses standardized correlations as the goal variable.
The outcome of standardized correlations is contrasted towards the mutual data technique, which uses average squared mistakes to calculate correlations.

\section{Proposed Method}

The overall course of the picture enrollment process is depicted in Figure 1. 2 Mri scans are used in the imaging enrollment process: a source images IR, as well as a scenario picture, IS that must be synchronized with the point cloud.

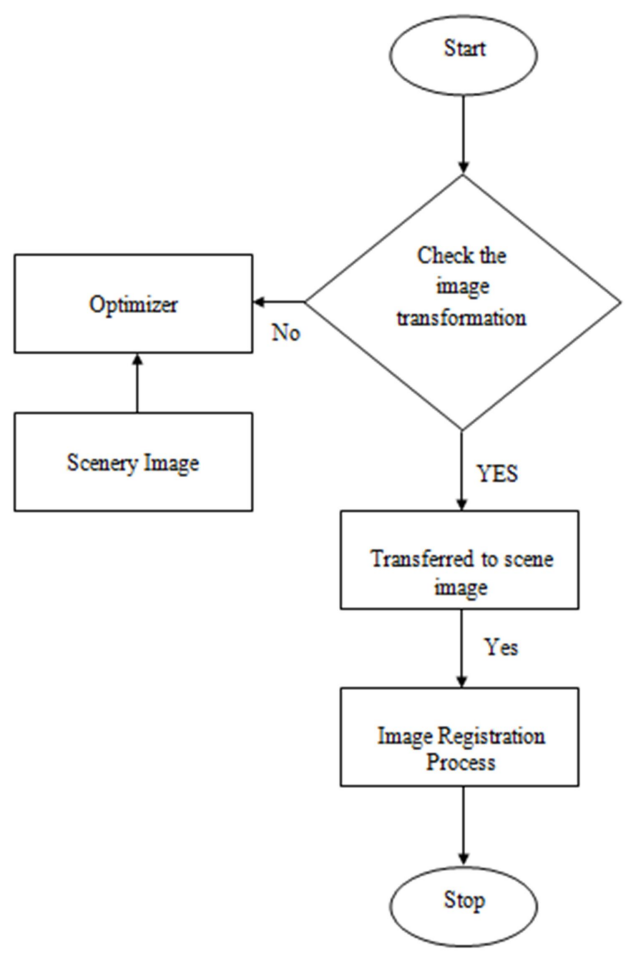

Figure 1: Flow diagram of the image registration procedure

This standard IR as well as converted picture is subjected to comparable scenario picture $\mathrm{f}(\mathrm{IS})$ were comparable as an modifications, such as translating, rotations, outcome of the enrollment process. A few of as well as scale. This relating to ", those two pictures are turned to $180^{\circ}$ and standardized correlations, is used to compute used as the captured images. The scenario the similarity here between standard with 
modified image sequences. Standardized covariance (NC) is established when $X(a, b) \& Y(a, b)$ were two pictures.

\section{Normalised Correlation}

$$
=\frac{1}{m} \sum_{a, b} \frac{(X(a, b)-\bar{X})}{\sigma_{X}} \frac{(Y(a, b)-\bar{Y})}{\sigma_{Y}}
$$

Where $\mathrm{m}=$ number of pixels in two images, $\bar{X}, \bar{Y}$ are average intensity values.

\section{Genetic Algorithm}

Whereas if the optimum conversion cannot be found, an ant colony optimization algorithm is employed to find the best one. This optimum conversion is found using evolutionary algorithms. A format string approach [7] was utilized as the research methodology. Encoding the characteristics within GA chromosomal is a vital step in GA. Instead of binary strings, a genuine compression algorithm is utilized. Because there is no translation to digital kind thus various genes operators may be utilized, the effectiveness of GA is enhanced [11]. As a result, the conversion variables are actively engaged in the coding. It's made up of five actual figures: one rotating variable $(\tau)$, two translational variables $(\mathrm{t})$, plus two scalability variables (s). The GA cycle consists of four steps: health assessment, choice, mixing, \& community formation. The starting population has 50 genomes. When discovering the likeness with each mutation, a standardized correlation is employed, as well as the performance index of such an optimization problem is computed for every chromosomal inside the community. The next stage is to choose the fitness values. Because the championship technique takes longer to execute, the roulette table approach is employed. Candidates with the best match were chosen, then optimization algorithms like evolutionary algorithms are used to generate the next generational community. Mathematical crossovers are employed in this case.

$$
\begin{gathered}
A=\mu * X+(1.0-\mu) * Y \\
B=(1.0-\mu) * X+\mu * Y
\end{gathered}
$$

Where X, Y - individual from the old generation.

As a result, both children, as well as the strongest people throughout prior eras, would be found in subsequent generations. Mutations operations choose one of the chromosome's individuals at arbitrary \& substitute it with a probability variable out of its spectrum. Again for the following generation of young people, a performance price is determined. That cycle has been repeated for so many decades. The highest production was selected as the terminating condition. Optimal endurance, i.e. best metamorphosis, is attainable as generations rise. The procedure is iterated again until 
endurance value exceeds a good enough level to finalize the certification. This optimal solution is attained by forming a new community from current people.

\section{Analysis}

Simulated real-world MRI samples were captured from a searchable database for this study. Images were acquired through McGill School's cerebral web database, which could be used to quickly assess the effectiveness of different image processing techniques. The biomedical scientific community had regularly utilized it. The pictures were obtained either with or without sounds, as well as with and without wounds. The pictures have a resolution of 181 X 217 dots each.

Here on a collection of pictures, modification variables are performed. The translational, rotations and scalability parameters were denoted by the letters tx, ty,
Sx, and Sy, accordingly. Scales was [0.5 2], rotations is [0 to 2], translations is [100 300], and rotations is $[0$ to 2]. The initial population was set at 50 people. The likelihood of crossovers is 0.6 , while the chance of a mutant is 0.3 . This maximal production is chosen as the terminating condition. A total of 300 cycles had been completed.

\section{Observations}

A total of two sets of MRI pictures were collected. GA was done with NC plus data fusion for each pairing of pictures (MI). The similarity measure is used to determine efficiency when employing NC. A maximum of 10 runs was performed with each iteration. The resulting findings were calculated using the mean-square method (MSE). The GA involving $\mathrm{NC}$ resulted in an improved outcome than that of the GA with MI.

Table 1: Images that were used

\begin{tabular}{|c|c|c|}
\hline Cases & Picture as a guide & Picture of a scenery \\
\hline A & Average & $\mathbf{1 8 0}$ degree \\
\hline B & Average & $\mathbf{5 \%}$ Noise \\
\hline C & Average & $\mathbf{3 \%}$ Noise \\
\hline D & Average & $\mathbf{1 \%}$ Noise \\
\hline
\end{tabular}

Table 2: The GA-based NC optimization outcome

\begin{tabular}{|c|c|c|c|c|}
\hline Cases & Normalized Correlation FV & Spin & Traduction & Scalability \\
\hline A & $\mathbf{2 . 5 6}$ & 4.56 & $\mathbf{1 8 5 . 5 6 1 4 3 . 0 0}$ & {$[22]$} \\
\hline B & 2.58 & 7.82 & 186.36186 .23 & {$[22]$} \\
\hline C & 2.53 & 7.81 & 185.67135 .28 & {$[22]$} \\
\hline D & 2.42 & 7.34 & 185.53183 .00 & {$[22]$} \\
\hline
\end{tabular}



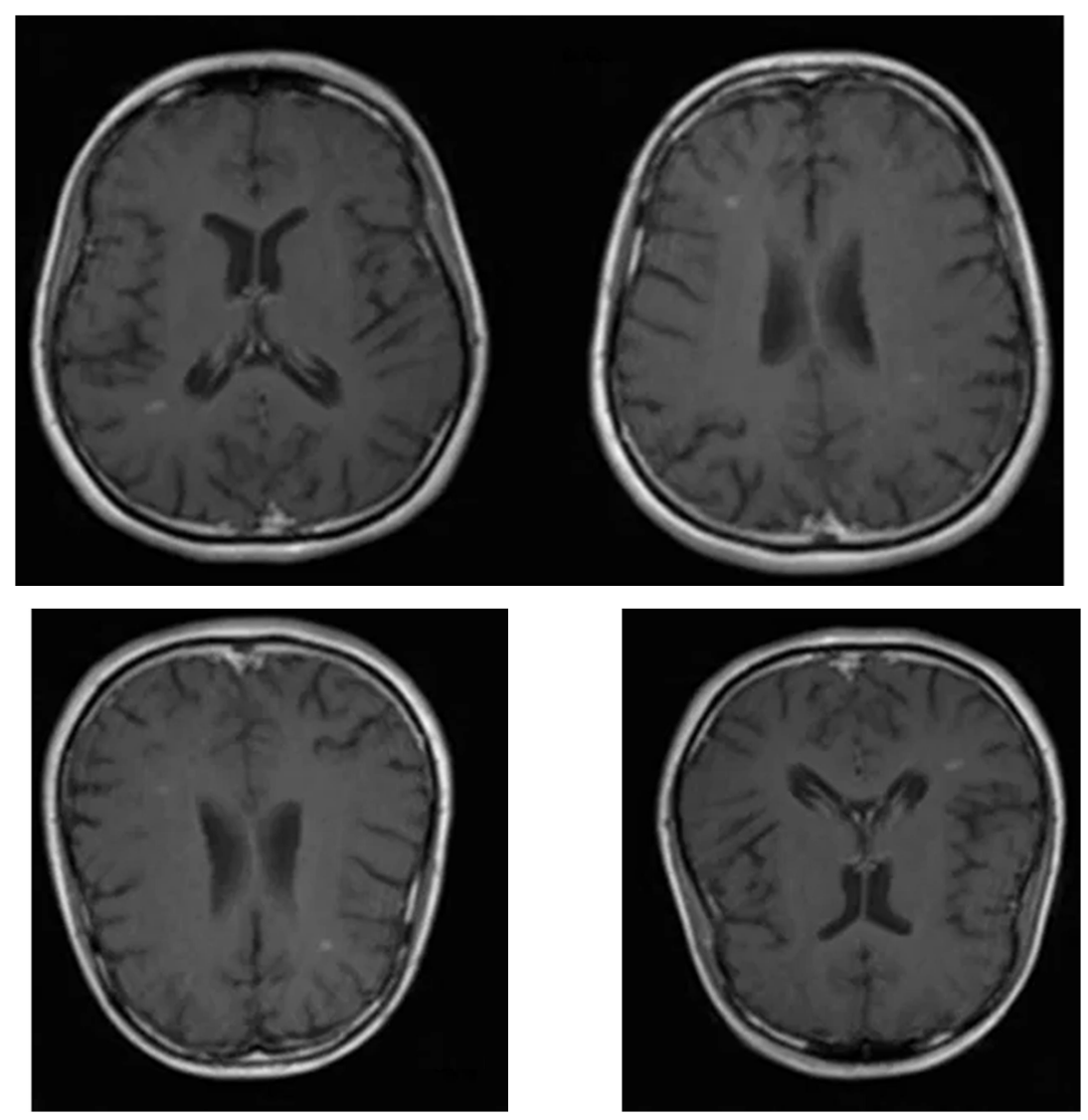

Figure 2: It represents a 180-degree rotation of the reference and scene picture using NC and MI

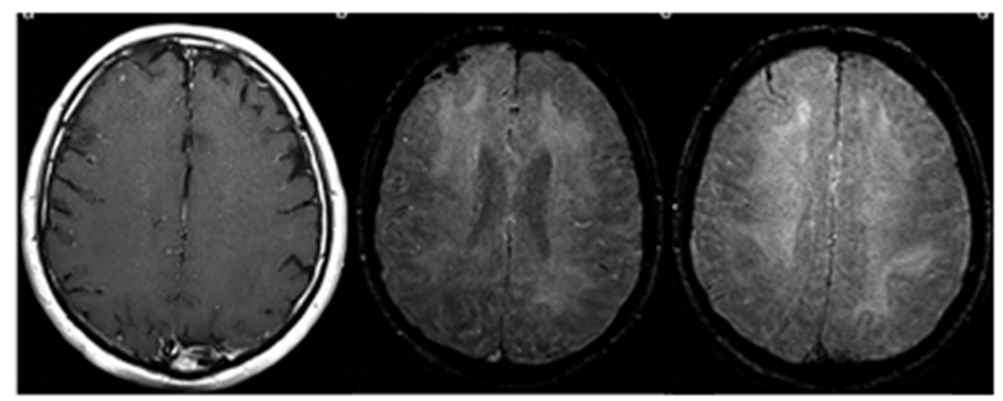

Figure 3: It represents a 180-degree rotation of the reference and scene picture using NC and MI with Noise

Table 3: The GA-based MI optimization outcome

\begin{tabular}{|c|c|c|c|c|}
\hline Cases & Mutual Information & Spin & Traduction & Scalability \\
\hline A & 2.86 & 4.89 & 156.00153 .32 & {$[22]$} \\
\hline B & 2.26 & 7.85 & 153.00135 .26 & {$[22]$} \\
\hline C & 2.86 & 7.95 & 157.32123 .79 & {$[22]$} \\
\hline D & 2.85 & 7.62 & 153.21123 & {$[11]$} \\
\hline
\end{tabular}

Table 4: NC versus MI using MSE

\begin{tabular}{|c|c|c|}
\hline Cases & $\begin{array}{c}\text { Normalized Correlation-Mean } \\
\text { Squared Root }\end{array}$ & $\begin{array}{c}\text { Mutual Information - Mean } \\
\text { Squared Root }\end{array}$ \\
\hline A & 9.23 & 11.89 \\
\hline B & 9.72 & 12.26 \\
\hline C & 9.38 & 10.37 \\
\hline D & 9.37 & 11.52 \\
\hline
\end{tabular}




\section{CONCLUSION}

Whenever contrasted to $\mathrm{MI}$, it could be observed that standardized correlations demonstrated greater converging and also had a lower MSE score in both situations. It's been found that as the number of generations grows, so does the fitness value. The converging is greater when the performance score is greater and much more consistent. Using standardized correlations, data fusion, and average error propagation methods were being used to analyze the optimization methods for GA. This standardized correlations technique performs superior to another. Registering is nothing more than optimization of mutual information directly derived from the pixel intensities of the pictures, which results in the computation of an image enhancement $T$. The effectiveness of intensity-based \& feature-based approaches might well be examined in future research.

\section{REFERENCES}

[1] Narasimhan SG, Nayar SK. Contrast restoration of weather degraded images. IEEE transactions on pattern analysis and machine intelligence. 2003 Jun 5; 25(6): 713-24.

[2] Fayyad J, Jaradat MA, Gruyere D, Najjaran H. Deep learning sensor fusion for autonomous vehicle perception and localization: A review. Sensors. 2020 Jan; 20(15): 4220.

[3] Osco LP, de Arruda MD, Junior JM, da Silva NB, Ramos AP, Moryia ÉA, Imai NN, Pereira DR, Creste JE, Matsubara ET, Li J. A convolutional neural network approach for counting and geolocating citrus-trees in UAV multispectral imagery. ISPRS Journal of Photogrammetry and Remote Sensing. 2020 Feb 1; 160: 97-106.

[4] Hu JW, Zheng BY, Wang C, Zhao CH, Hou XL, Pan Q, Xu Z. A survey on multi-sensor fusion based obstacle detection for intelligent ground vehicles in off-road environments. Frontiers of Information Technology \& Electronic Engineering. 2020 May; 21: 675-92.

[5] Punn NS, Agarwal S. Modality specific U-Net variants for biomedical image segmentation: A survey. arXiv preprint arXiv:2107.04537. 2021 Jul 9.

[6] Imani M, Hassan EB, Duque G. Validation of a Biomedical Quantitative Image Analysis Software for Musculoskeletal Diseases. In Osteoporosis International 2020 Dec 1 (Vol. 31, No. SUPPL 1, pp. S499S501). 236 Grays Inn RD, $6^{\text {th }}$ Floor, 
London WC1X 8HL, England: Springer London Ltd.

[7] Luca AR, Ursuleanu TF, Gheorghe L, Grigorovici R, Iancu S, Hlusneac M, Preda C, Grigorovici A. Designing a High-Performance Deep Learning Theoretical Model for Biomedical Image Segmentation by Using Key Elements of the Latest U-Net-Based Architectures. Journal of Computer and Communications. $2021 \mathrm{Jul}$ 2;9(7):8-20.

[8] Anilkumar KK, Manoj VJ, Sagi TM. A survey on image segmentation of blood and bone marrow smear images with emphasis on automated detection of Leukemia. Biocybernetics and Biomedical Engineering. 2020 Sep 11.

[9] Chakraborty S, Mali K. An overview of the biomedical image analysis from the deep learning perspective. Applications of Advanced Machine Intelligence in Computer Vision and Object Recognition: Emerging Research and Opportunities. 2020: 197-218.

[10] Xiao P, Haque E, Zhang T, Dong $\mathrm{XN}$, Huang Y, Wang X. Can DXA image-based deep learning model predict the anisotropic elastic behavior of trabecular bone? Journal of the Mechanical Behavior of Biomedical Materials. 2021 Sep 15:104834.

[11] Kuppala K, Banda S, Barige TR. An overview of deep learning methods for image registration with a focus on feature-based approaches. International Journal of Image and Data Fusion. 2020 Apr 2; 11(2): 113-35.

[12] Kumawat A, Panda S. A robust edge detection algorithm based on feature-based image registration (FBIR) using improved canny with fuzzy logic (ICWFL). The Visual Computer. 2021 Jul 14: 1-22.

[13] Kist AM, Zilker J, Döllinger M, Semmler M. Feature-based image registration in structured light endoscopy. Medical Imaging with Deep Learning 2021 Feb 10.

[14] Lu J, Jia H, Li T, Li Z, Ma J, Zhu R. An Instance Segmentation-Based Framework for a Large-Sized HighResolution Remote Sensing Image Registration. Remote Sensing. 2021 Jan; 13(9): 1657.

[15] Zheng Y. Global optimal solution on blending problem. InJournal of Physics: Conference Series 2021 
May 1 (Vol. 1914, No. 1, p. 012048). IOP Publishing.

[16] Yang F, Sun Q, Jin H, Zhou Z. Superpixel segmentation with fully convolutional networks.
InProceedings of the IEEE/CVF Conference on Computer Vision and Pattern Recognition 2020 (pp. 13964-13973). 